\title{
Left Ventricle Non-Compaction Myocardium and Thrombophilia in a Pregnant Woman
}

\author{
Elena A. Kochmareva ${ }^{1}$, Valentin A. Kokorin ${ }^{1}$, Evgenia A. Kondrashova ${ }^{2}$, Nadejda V. Khokhlova ${ }^{2}$, \\ Argishti G. Vardanyan ${ }^{1}$, Ilya A. Kokorin ${ }^{1}$, Dmitry A. Doroshenko² \\ ${ }^{1}$ Hospital Therapy No. 1 Department, Pirogov Russian National Research Medical University, Moscow, Russian Federation \\ ${ }^{2}$ City Clinical Hospital No. 15 named after O.M. Filatov, Moscow, Russian Federation
}

Received: 30/03/2016

Accepted: 02/05/2016

Published: 22/06/2016

How to cite this article: Kochmareva EA, Kokorin VA, Kondrashova EA, Khokhlova NV, Vardanyan AG, Kokorin IA, Doroshenko DA. Left ventricle noncompaction myocardium and thrombophilia in a pregnant woman. EJCRIM 2016;3:doi:10.12890/2016_000432

Conflicts of Interests: The Authors declare that there are no competing interests.

This article is licensed under a Commons Attribution Non-Commercial 4.0 License

\section{ABSTRACT}

Non-compaction of the ventricular myocardium (NCM) is a genetic cardiomyopathy usually due to mutationof the G4.5 gene located in the $\mathrm{Xq} 28$ chromosomal region. This congenital disorder is characterized by pronounced trabeculations and intertrabecular recesses resulting from abnormal embryogenesis between the fifth and eighth fetal weeks. The reported prevalence in the general population is between $0.014 \%$ and $1.3 \%$. The classic triad of complications includes heart failure, ventricular arrhythmias and systemic embolic events, although some patients have an asymptomatic form. NCM is commonly diagnosed by echocardiography, but contrast ventriculography, CT and MRI can also be used. Here we present a case of left ventricle NCM, manifested after respiratory infection, in a pregnant patient with congenital thrombophilia and a history of myocardial infarction.

\section{LEARNING POINTS}

- Non-compaction myocardium (NCM) in pregnant women has been associated with a poor prognosis.

- We should avoid routinely recommending young women with NCM to refuse pregnancy.

- A decision to continue pregnancy should be made by the patient in discussion with specialists.

\section{KEYWORDS}

Non-compaction myocardium, pregnancy, heart failure, thrombophilia.

\section{CASE REPORT}

A 26-year-old female patient was admitted to hospital in 2014 with complaints of dyspnoea. The patient had a family history of sudden death (her father), but she herself had shown no signs of cardiovascular disease until 2009. Pregnancies in 2007 and 2008 had ended in miscarriage at 6-8 weeks. Antiphospholipid syndrome was excluded. In 2009, signs of heart failure (shortness of breath, oedema of the legs) and episodes of unsustainable ventricular tachycardia (VT) manifested after respiratory infection and were associated with a reduction in ejection fraction (EF) to 26\%. In 2010 the patient had a myocardial infarction of the LV inferior wall and right ventricle (RV), complicated by thrombus formation in the RV. Intact coronary arteries were found on angiography. Embolic genesis of the myocardial infarction was suspected. Genetic testing revealed hereditary thrombophilia with increased sensitivity to warfarin. Echocardiography and MRI were performed and non-compaction myocardium of the LV was observed (Figs. 1 and 2). Following therapy with ACE inhibitors, beta-blockers, 
diuretics, digoxin and warfarin, the patient's condition stabilized: signs of heart failure and rhythm disorders regressed and EF increased to $46 \%$.

In 2014, the patient became pregnant despite the high risk of a poor outcome for her and her fetus. She was admitted to hospital at 1819 weeks of gestation. Routine blood tests were normal. However, proBNP was 182 ng/ml (normal 0-100 ng/ml) and EF was 36\% on echocardiography (Table 1). Nevertheless, her condition and that of the fetus was completely stable and the expert consensus opinion was to continue the pregnancy in line with her choice also.

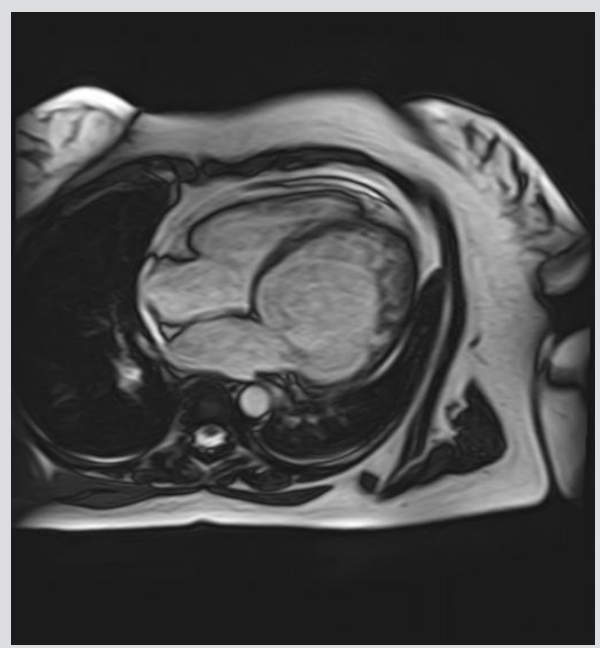

Figure 1: Pronounced trabeculations and intratrabecular recesses in the apex of the left ventricle, visualized by MRI and indicating non-compaction myocardium.

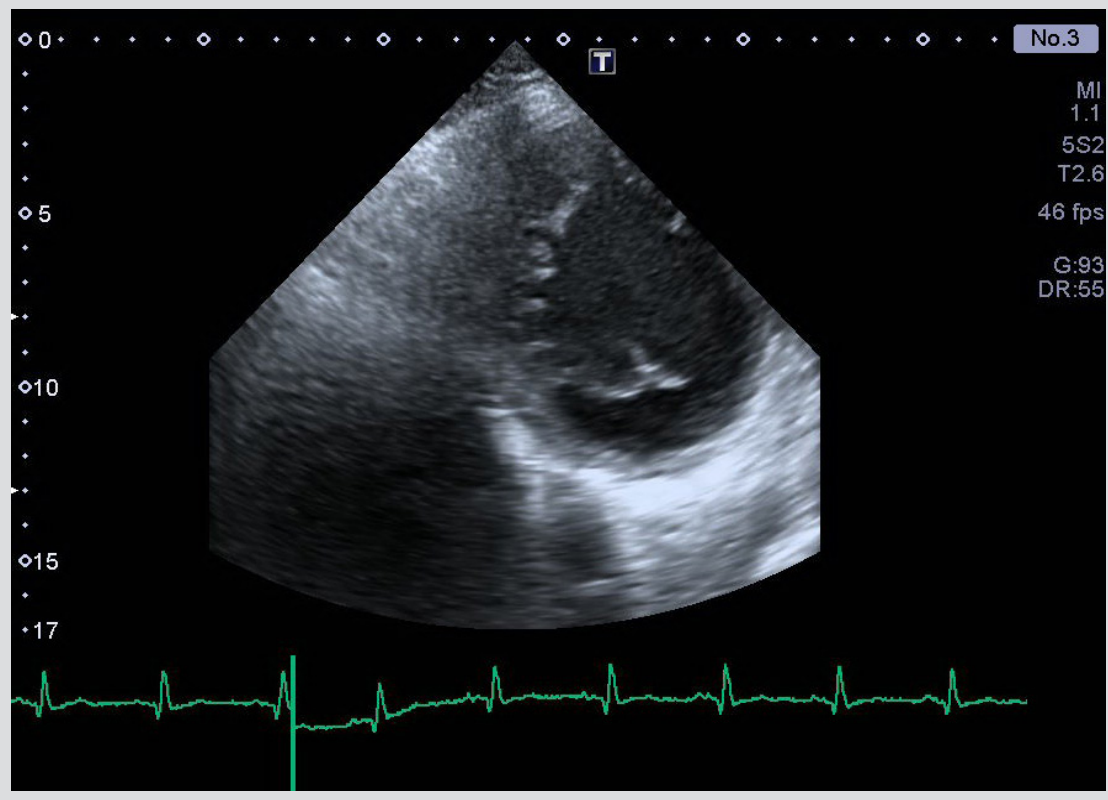

Figure 2: Pronounced trabeculations and intratrabecular recesses in the intraventricular septum, visualized by echocardiography. A short-axis view of the left ventricle at the level of the mitral valve is shown.

The patient took fraxiparine subcutaneously (under anti-Xa-factor control), beta-blockers and diuretics. The patient was discharged with a recommendation to present for follow-up at 28 weeks of pregnancy. She was readmitted to the cardiology department for the second time at 26 weeks of gestation with complaints of worsening dyspnoea. EF had decreased to $23 \%$ (Figs. 3 and 4 ) and proBNP was $462 \mathrm{ng} / \mathrm{ml}$. The dosage of beta-blockers and diuretics was corrected and the patient was discharged from hospital at her own request.

At 31 weeks of pregnancy, the patient was hospitalized again with orthopnoea, an extremely low EF (20\%) and stage 1 pulmonary hypertension. Episodes of unstable VT were seen. Due to her worsening condition, a caesarean section was performed and the baby was 
born alive. Because of the high risk of life-threatening arrhythmias, a cardioverter-defibrillator was implanted in the patient.

The patient was observed 8 months after childbirth. She had no complaints and echocardiography showed that EF had increased to $32 \%$ (Table 1). The infant had no signs of cardiovascular disease and no pathology on echocardiography.

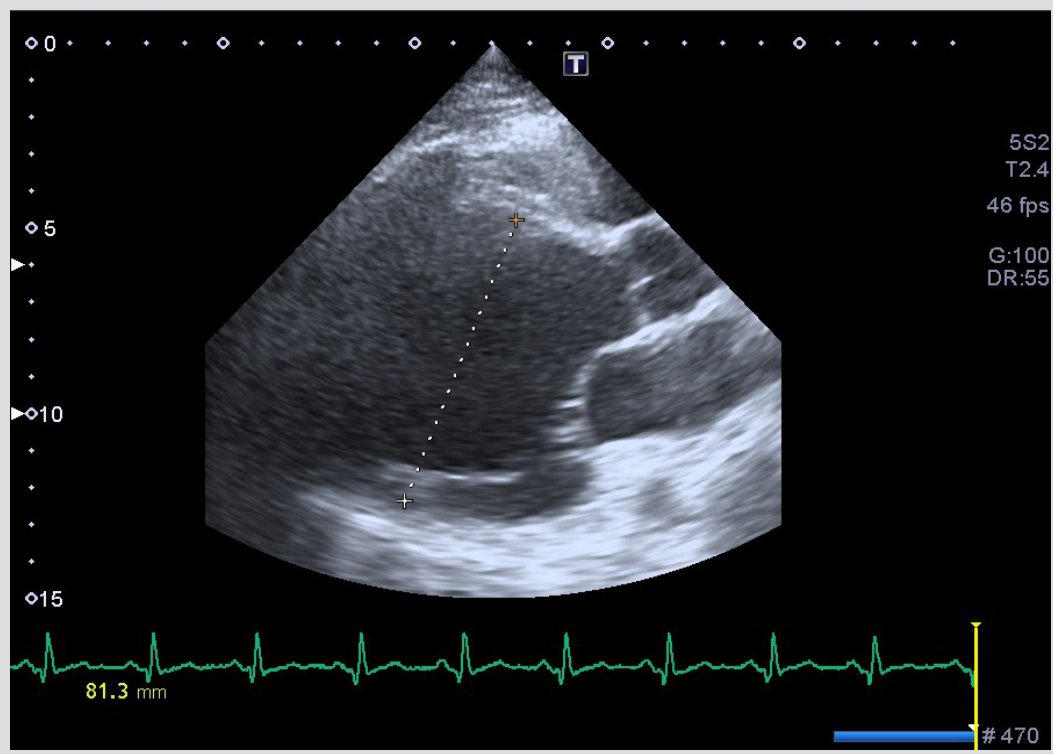

Figure 3: Long-axis view, parasternal position showing severe dilatation of the left ventricle (end-diastolic dimension $81.3 \mathrm{~mm}$ )

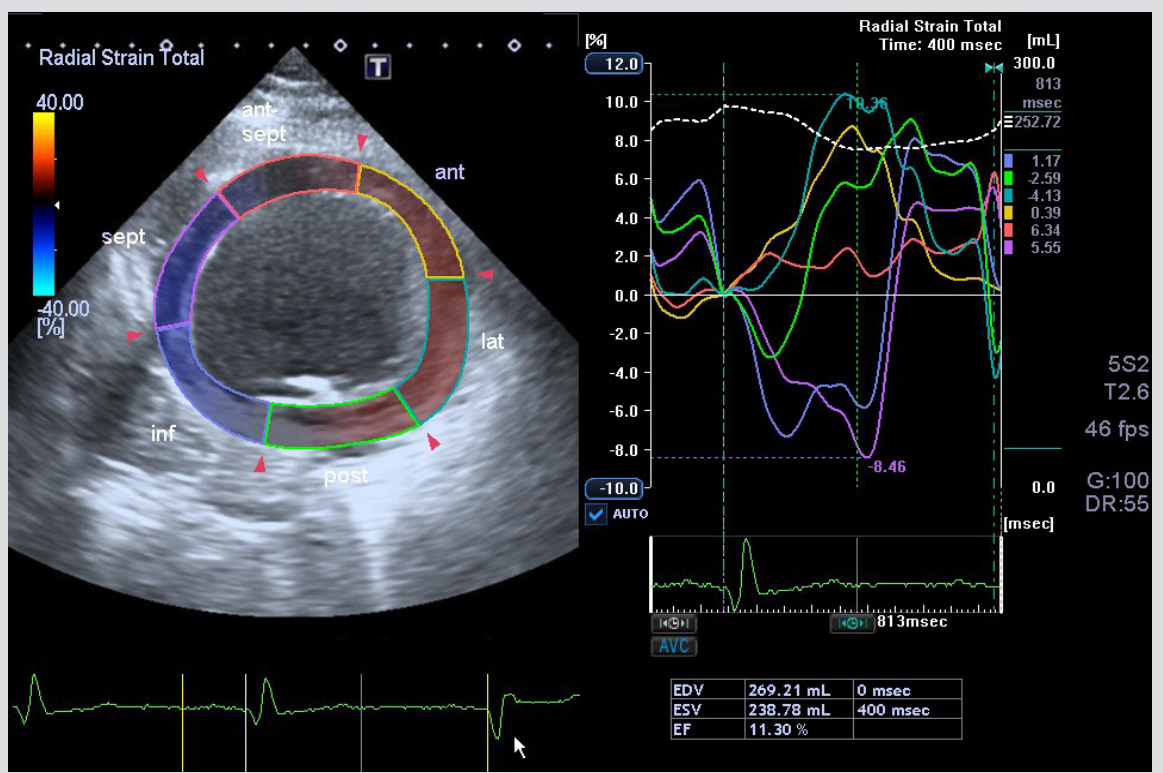

Figure 4: Short-axis view, parasternal position at the level of the mitral valve, showing a decrease in radial systolic left ventricle deformation parameters caused by dyssynchrony of the inferior wall and inferior part of the intraventricular septum (blue and purple lines in the diagram).

\section{DISCUSSION}

There are a few cases in the literature describing patients with NCM and pregnancy. NCM has been associated with a poor prognosis and high incidence of complications, such as heart failure, ventricular tachyarrhythmias and thromboembolism. Our patient had all these 
complications, but she decided to continue her pregnancy despite the very high risk to her life. In most cases patients become symptomatic during last trimester of pregnancy because of haemodynamic changes, and the question of the method of delivery arises. This decision should be taken by the clinicians keeping in mind the possible risks of different methods of delivery for the mother and fetus. It is important to monitor the patient's status, symptoms of congestive heart failure, LV ejection fraction and BNP as well as indicators of blood coagulation for appropriate decision-making concerning the timing of delivery. We have described a favourable pregnancy outcome in a patient with two hereditary disorders. This case shows that physicians should not underestimate the long-term complications, especially persistent progressive heart failure. However, despite the high risk of a poor outcome in this population, we should avoid routinely recommending young women to refuse pregnancy.

\begin{tabular}{|l|l|l|l|l|l|l|l|l|l|}
\hline & LA, mm & EDV, $\mathrm{ml}$ & ESV, $\mathrm{ml}$ & LVEF, \% & $\begin{array}{l}\text { SPPA, } \\
\mathrm{mmHg}\end{array}$ & MR & TR & RA, mm & RV, $\mathrm{mm}$ \\
\hline $\begin{array}{l}18^{\text {th }} \text { week } \\
\text { of gestation }\end{array}$ & $39 \times 46$ & 201 & 151 & 36 & 29 & 1 & $0-1$ & 38 & 30 \\
\hline $\begin{array}{l}\text { 8 months after } \\
\text { caesarean } \\
\text { section }\end{array}$ & $46 \times 59$ & 285 & 195 & 32 & 25 & $2-3$ & $0-1$ & 40 & 30 \\
\hline
\end{tabular}

The patient's condition improved and dyspnoea regressed after the caesarean section despite worsening echocardiography parameters (dilatation of the left chambers), with no significant changes in ejection fraction or pulmonary artery pressure.

EDV: end-diastolic volume; ESV: end-systolic volume; LA: left atrium; LVEF: left ventricle ejection fraction; MR: mitral regurgitation; SPPA: systolic pulmonary artery pressure; $T R$ : tricuspid regurgitation; RA: right atrium; $R V$ : right ventricle.

\section{REFERENCES}

1. Nikolaeva MG, Grigorieva EE, Serduk GV. Noncompaction of the left ventricular myocardium and pregnancy. Mother and Child in Kuzbass 2014;2:159-162 [in Russian].

2. Kilic ID, Tanriverdi H, Evrengul H, Uslu S, Sungur MA. Left ventricular non-compaction in pregnancy. Cardiovasc J Afr 2013;24:e1-2.

3. Plastiras SC, Pamboucas C, Toumanidis S. Noncompaction cardiomyopathy and pregnancy: an alarming coexistence ending in a favourable outcome. Exp Clin Cardiol 2012;17:136-138.

4. Sawant RD, Freeman LJ, Stanley KPS, McKelvey A. Pregnancy and treatment outcome in a patient with left ventricular non-compaction. Eur J Heart Fail 2013;15:592-595. 